\title{
Evaluating the Effect of Oprelvekin on Cardiac Repolarization in Subjects with Chemotherapy-Induced Thrombocytopenia: An Observational Chart Review of a Phase 2 Clinical Trial in Laredo, Texas
}

\author{
Soriano $\mathrm{RA}^{* 1}$ and Gonzalez $\mathrm{M}^{2}$ \\ ${ }^{1}$ The London School of Economics and Political Science, London, UK \\ ${ }^{2}$ Envision Clinical Research, LLC, Laredo Texas 78041, USA
}

*Corresponding author: Soriano RA, The London School of Economics and Political Science, London, UK, E-mail: R.A.Soriano@lse.ac.uk

Citation: Soriano RA, Gonzalez M (2017) Evaluating the Effect of Oprelvekin on Cardiac Repolarization in Subjects with Chemotherapy-Induced Thrombocytopenia: An Observational Chart Review of a Phase 2 Clinical Trial in Laredo, Texas. J Case Rep Stud 5(2): 204. doi: 10.15744/2348-9820.5.204

Received Date: March 05, 2017 Accepted Date: April 26, 2017 Published Date: April 28, 2017

\begin{abstract}
This study tested for the cardiac effects of Oprelvekin, recombinant human interleukin-11, a thrombopoietic growth factor, in patients afflicted with chemotherapy-induced thrombocytopenia (platelet count $<50,000$ cells/ul). Chart-reviews of patients fulfilling the inclusion criteria of: 18-75 yrs of age, non-myeloid malignancy, with adequate hematologic, hepatic and renal parameters and normal electrocardiograms that were enrolled in this phase 2 trial were analyzed. Patients of child-bearing potential agreed to be on a reliable form of birth control for the duration of the study. Results on 4 patients suggest that Oprelvekin does not cause atrial nor ventricular arrhythmia, a rare severe cardiac side effect, in treating patients with chemotherapy-induced thrombocytopenia.
\end{abstract}

Keywords: Neumega; Chemotherapy-Induced Thrombocytopoenia; Oprelvekin

List of Abbreviations: rIL-11: Recombinant Interleukin-11; FDA: US Food and Drug Administration; mcg: microgram; ECG: Electrocardiogram; PK: Pharmacokinetic; SC: Subcutaneous

\section{Introduction}

Thrombocytopenia is a common occurrence in cancer patients [1-4]. Albeit affecting small numbers annually, it may develop into a serious condition, causing internal bleeding, decreasing chemotherapy efficacy, and in a few cases, death [2]. Additionally, chemotherapy treatment may be reduced to insufficient levels or even delayed because of thrombocytopenia [2]. There are multiple causes of thrombocytopenia in cancer: chemotherapy-induced, immune reaction, coagulopathy, infection, drug reaction, post-transfusion purpuric thrombocytopenia. Chemotherapy is the most frequent cause [1,4]. Within chemotherapy-induced thrombocytopenia, there are variations in rates in conjunction with various chemotherapy treatments [1]. Platelet transfusion is the primary treatment modality for chemotherapy-induced thrombocytopenia. However, it is not without risks. The risks for infectious and immunologic complications increases as the number of transfusions administered [2].

Oprelvekin, recombinant human IL-11, is a pleiotropic hematopoietic growth factor that stimulates production, differentiation, and maturation of progenitors of the myeloid, erythroid, megakaryocyte, and lymphoid cell lines [5-8] (Figures 1 and 2) [8]. FDA-approved in the United States since 1997, it is used to prevent severe chemotherapy-induced thrombocytopenia. Using recombinant DNA technology, the active ingredient is derived from Escherichia coli. In adults, it is used to reduce the need for platelet transfusions following myelosuppressive chemotherapy with nonmyeloid malignancies [3-5,7,9]. Estimates of overall cost of administration of platelet transfusion and Oprelvekin appear to be almost comparable.

Tachycardia, pleural effusion, dilutional anemia, and atrial arrhythmias have been reported as adverse events in adults [5,9]. The safety and efficacy of Oprelvekin has not been established in children. Increases in fluid retention and plasma volume are known root causes of the pulmonary and cardiac [9-11]. They are usually reversible and treated with use of diuretics, low sodium diets [12] and discontinuation of medication [9]. Most of these adverse events are of mild or moderate severity. Patients with a history of arrhythmias are cautioned on the use Oprelvekin. Previous clinical trials documented a 15\% occurrence of brief atrial arrhythmias in patients treated with a dose of $50 \mathrm{mcg} / \mathrm{kg}[6,12]$. To date, the mechanism of induction of arrhythmias is unknown [6,12]. Ventricular arrhythmias, though rarely, have been reported up to 1 week after initiation of treatment of Oprelvekin [6,13,14]. 


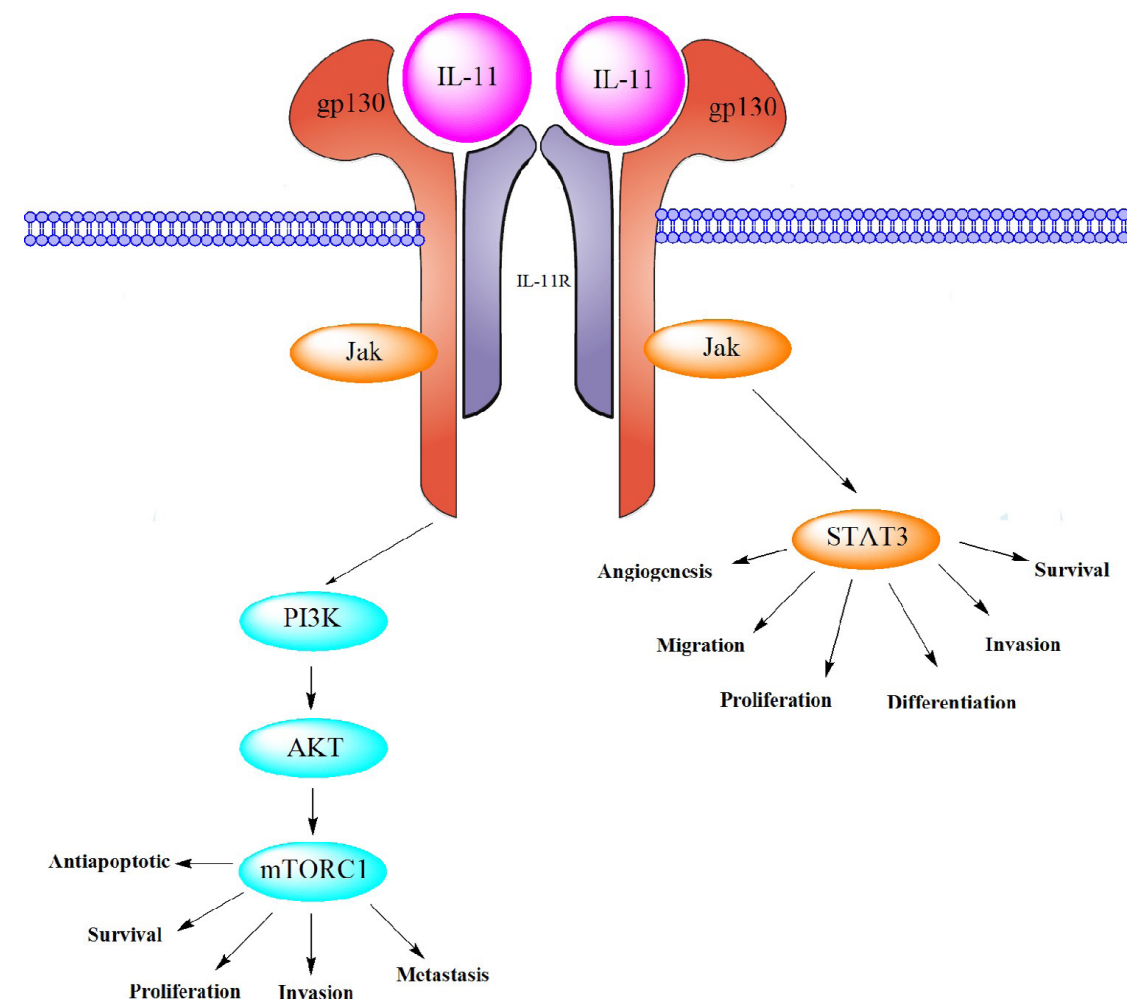

Figure 1: IL-11 Signal Pathway (From Zhu Z, Fang Y, Wakefield M: The role of IL-11 in immunity and cancer: Cancer Letters, January 2016, p157)

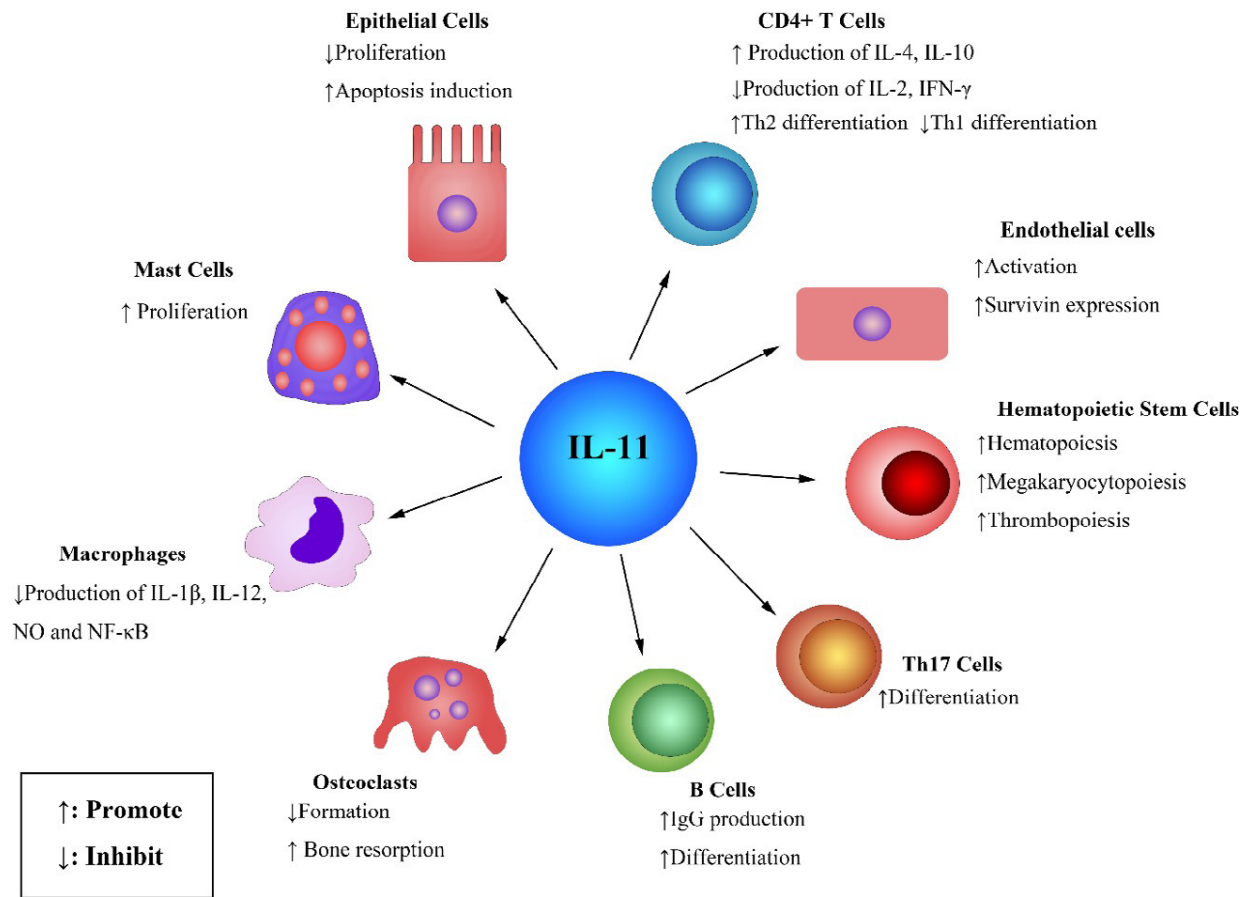

Figure 2: The effects of IL-11 on various cells (From Zhu Z, Fang Y, Wakefield M: The role of IL-11 in immunity and cancer: Cancer Letters, January 2016, p159)

This retrospective chart review reviewed for potential cardiac adverse event or toxicity on four patients who received treatment with $50 \mathrm{mcg} / \mathrm{kg}$ subcutaneously of Oprelvekin for their chemotherapy-induced thrombocytopenia following a global study protocol $[6,13,14]$.

\section{Methodology}

This single-arm study was part of a phase 2, open-label, pharmacokinetic and pharmacodynamic multicenter, study in subjects nonmyeloid malignancies whose chemotherapy has induced thrombocytopenia (defined as platelet count $<50,000$ cells/ul) $[6,13,14]$. Subjects and investigators had knowledge of Oprelvekin treatment. 
Baseline vital signs were obtained with each study visit. Hematology and blood chemistries were obtained on screening day to determine eligibility; 12-lead Electrocardiograms (ECGs) in triplicate predose and subsequently were done as per study protocol. Oprelvekin at a dose of $50 \mathrm{mcg} / \mathrm{kg}$ once daily was administered by subcutaneous (SC) injection within 6-24 hours of completion of chemotherapy. The minimum treatment duration is 3 continuous days and maximum duration of 21 days of treatment. Total duration of the study was determined by the physician until the desired platelet response was achieved. Test drug was administered by study staff on the first and third visits; the subject self-administered on day 2 of the study. The physician determined whether the subjects were capable of self-administering daily Oprelvekin. Subjects who presented with abnormal laboratory values were evaluated further to determine the etiology. Abnormal liver and renal function tests were categorized as serious adverse events. The end of the study was marked by the final study visit, approximately 14 days after the last Oprelvekin dose.

There were 54 patients recruited globally. Four [4] eligible subjects in Laredo, Texas met all inclusion criteria and none of the exclusion criteria (Table 1). These four subjects remained eligible throughout the study and maintained compliance with the study protocol. No one took any medication that could have affected the QT/QTc prolongation as listed in www.QTdrugs.org [15]. Tobacco, caffeine and alcohol were avoided. No adverse events were reported up to 28 days from the last dose of the study medication.

\begin{tabular}{|c|c|}
\hline Inclusion Criteria & Exclusion Criteria \\
\hline $\begin{array}{l}\text { 1. Men and women ages } 18 \text { to } 65 \text { with nonmyeloid malignancies } \\
\text { for whom Oprelvekin is indicated. } \\
2 \text {. All female and male subjects who are biologically capable of } \\
\text { having children must agree and commit to the use of a reliable } \\
\text { method of birth control for the duration of the study and for } \\
\text { one month after the last dose of test article. } \\
\text { 3. At least one documented occasion of adequate hematologic } \\
\text { recovery from previous of current chemotherapy, defined by the } \\
\text { presence of all of the following: } \\
\text { a. Absolute neutrophil count (ANC) } \geq 1,000 \text { cells/ul } \\
\text { b. Platelet count } \geq 75,000 \text { cells/ul } \\
\text { c. Hemoglobin concentration }>9 \mathrm{~g} / \mathrm{dl} \text { (transfusion to this level } \\
\text { allowed) } \\
4 \text {. Adequate renal and hepatic excretory function, defined as } \\
\text { both serum creatinine and total bilirubin levels } \leq 1.5 \text { times the } \\
\text { upper limit of normal. } \\
5 \text {. Normal baseline electrocardiogram. }\end{array}$ & 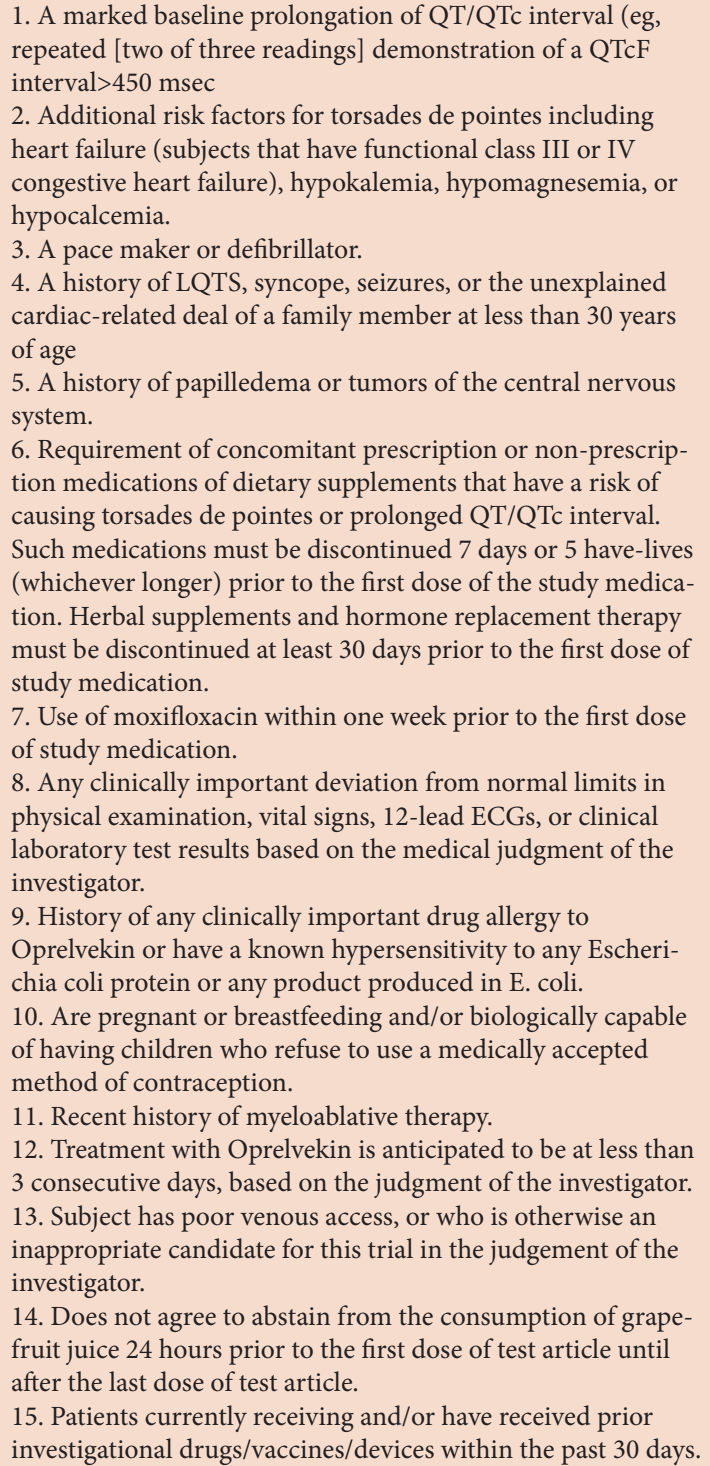 \\
\hline
\end{tabular}

Table 1: Inclusion and Exclusion Criteria

\section{Description of Study Flow Chart Protocol}

The administered test dose was based on weight submitted at the time of registration. The dose assigned at registration was administered on day 1, day 2 and day 3. Dosing beyond day 3 was administered (up to 21 days of treatment allowed in protocol) based on the medical judgment of the investigator. Height was measured at screening only (Table 2). 


\begin{tabular}{|c|c|c|c|c|c|c|c|c|c|}
\hline \multirow{3}{*}{$\begin{array}{c}\text { Study Phase } \\
\text { Study Day } \\
\text { Informed Consent }\end{array}$} & \multirow{3}{*}{$\begin{array}{c}\text { Screening } \\
-3 \\
\mathrm{X} \\
\end{array}$} & \multirow{3}{*}{$\begin{array}{c}\text { Predose } \\
-1\end{array}$} & \multicolumn{6}{|c|}{ Active Phase starting from first Oprelvekin dose till duration of treatment } & \multirow{3}{*}{$\frac{\text { Final Visit }}{5}$} \\
\hline & & & 1 & & & \multicolumn{3}{|c|}{ At least 3 Days of Treatment } & \\
\hline & & & & & & & & & \\
\hline Demographics & $\mathrm{X}$ & & & & & & & & \\
\hline Medical History & $\mathrm{X}$ & & & & & & & & \\
\hline Physical Exam & $\mathrm{X}$ & & & & & & & & $\mathrm{x}$ \\
\hline Serum Pregnancy & $\mathrm{X}$ & & & & & & & & $\mathrm{x}$ \\
\hline Vital Signs & $\mathrm{X}$ & $\mathrm{X}$ & $\mathrm{X}$ & $\mathrm{X}$ & $\mathrm{X}$ & $\mathrm{X}$ & $\mathrm{X}$ & $\mathrm{X}$ & $\mathrm{X}$ \\
\hline Height and Weight & $\mathrm{X}$ & $\mathrm{X}$ & $\mathrm{X}$ & $\mathrm{X}$ & $\mathrm{X}$ & $\mathrm{X}$ & $\mathrm{X}$ & $\mathrm{X}$ & $\mathrm{X}$ \\
\hline 12-lead ECG (triplicate) & $\mathrm{X}$ & $\mathrm{X}$ & $\mathrm{X}$ & $\mathrm{X}$ & $\mathrm{X}$ & $\mathrm{X}$ & $\mathrm{X}$ & $\mathrm{X}$ & $\mathrm{X}$ \\
\hline Hematology \& Chemistry & $\mathrm{X}$ & $\mathrm{X}$ & $\mathrm{X}$ & $\mathrm{X}$ & $\mathrm{X}$ & $\mathrm{X}$ & $\mathrm{X}$ & $\mathrm{X}$ & $\mathrm{X}$ \\
\hline Urinalysis & $\mathrm{X}$ & $\mathrm{X}$ & & & & & & & \\
\hline Test Dose Administration & & & $\mathrm{X}$ & $\mathrm{X}$ & $\mathrm{X}$ & $\mathrm{X}$ & $\mathrm{X}$ & $\mathrm{X}$ & \\
\hline $\begin{array}{c}\text { Pharmacokinetic sample of } \\
\text { Oprelvekin }\end{array}$ & & & $\mathrm{X}$ & $\mathrm{X}$ & $\mathrm{X}$ & $\mathrm{X}$ & $\mathrm{X}$ & $\mathrm{X}$ & $\mathrm{X}$ \\
\hline $\begin{array}{l}\text { Monitoring for Other } \\
\text { Medications }\end{array}$ & $\mathrm{X}$ & $\mathrm{X}$ & $\mathrm{X}$ & $\mathrm{X}$ & $\mathrm{X}$ & $\mathrm{X}$ & $\mathrm{X}$ & $\mathrm{X}$ & $\mathrm{X}$ \\
\hline $\begin{array}{l}\text { Monitoring for Adverse } \\
\text { Events }\end{array}$ & $\mathrm{x}$ & $\mathrm{x}$ & $\mathrm{x}$ & $\mathrm{x}$ & $\mathrm{x}$ & $\mathrm{x}$ & $\mathrm{x}$ & $\mathrm{x}$ & $\mathrm{X}$ \\
\hline
\end{tabular}

Table 2: Study Flow Chart

The ECG or electrocardiogram was taken in triplicate, 1 to 2 minutes apart. The day 1 predose triplicate ECGs was conducted within 15 minutes before dosing at hour " 0 ". All post dose ECGs were taken within \pm 15 minutes of the scheduled timepoints; and the final ECGs were conducted within 3 days of the last dose of Oprelvekin. Subjects were instructed to rest in the supine position at least 10 minutes before the ECG was started. All ECGs were conducted on day 1 and conducted as close to the same clock times as the respective ECGs conducted on day -1. ECG timing was first priority in the conduct of the study. ECG and PK procedures had a mirrored relationship (i.e., if the ECG is 5 minutes late at hour 3 on day 1, then the PK sample for hour 3 on day 1 should be drawn 5 minutes late for that hour 3 draw). The primary endpoint was assessment of change in sample corrected QT interval $(\mathrm{QTcN})$.

Hematology and blood chemistry screening day laboratory results were used to determine eligibility. Hematology and blood chemistries were drawn within 1 hour prior to dosing on all required visits and coordinated on day 1 with the ECG and PK procedures in order to maintain the synchronization with predose day -1 ECG schedule. The protocol stated that if the laboratory evaluations before dose administration on Days 1 and 3 indicate that a subject's potassium, magnesium, or calcium level is below the lower limit of normal, that subject should not be dosed and is to be withdrawn from the study.

Day 1 test dose was administered 6 to 24 hours after the completion of chemotherapy. Treatment with Oprelvekin was to be discontinued at least 2 days before starting the next planned cycle of chemotherapy. Oprelvekin was administered via SC injection once daily until desired platelet response is attained which is $>50,000$ cells/ul (allowed up to 21 days of treatment) or based on the medical judgment of the investigator.

After ECG, pharmacokinetic sampling was next in priority in the conduct of the study. Serum collection of $4 \mathrm{~mL}$ of whole blood was obtained on Day 1 at " 0 " hour sample within 10 minutes before dosing for PK sampling. Blood was drawn within plus 5 minutes of the last of the corresponding triplicate ECGs at each scheduled timepoint. ECG and PK procedures had a relationship that was maintained (i.e., If the ECG is 5 minutes late at hour 3 on day 1, then the PK sample for hour 3 on day 1 should be drawn 5 minutes late for that hour 3 draw. Subsequent hour procedures for day 1 should revert back to the scheduled time to mirror day -1).

If the last dose of Oprelvekin occurred on any day other than days 1 or 3, a Final Study visit was conducted on the day of last dose. If the last dose of Oprelvekin was administered on days 1to 3, that day was considered the End of Treatment visit. If the subject has an unplanned last dose of Oprelvekin, the subject was scheduled for an End of Treatment or Final Study visit within 24 hours of the last dose. The minimum desired treatment duration is 3 continuous days. The maximum treatment duration is up to 21 days of treatment. The end of study visit was the final study evaluation conducted within 14 days after the final Oprelvekin dose. All doses of Oprelvekin administered during the study were recorded. Investigators were required to report any Adverse Events that occurred up to 14 days after the last dose of Oprelvekin, or after the last study observation, whichever occurs last, until the events have subsided, returned to baseline, or, in case of permanent impairment, until the condition stabilized.

\section{Results}

Oprelvekin was well-tolerated by $100 \%$ of subjects during the entire duration of the study. All four patient ECGs were consistently 
interpreted as normal, despite baseline analysis showing sinus tachycardia for all four study subjects. One subject with triplereceptor negative metastatic breast cancer developed thrombocytopenia after cycles of Gemcitabine and Carboplatin. The thrombocytopenia improved considerably with Oprelvekin, which was tolerated well. The other three study subjects had the non-myeloid malignancies of Ovarian Cancer, Prostate Cancer, and Colon Cancer. They similarly developed thrombocytopenia in their chemotherapeutic regimens and were administered Oprelvekin in 3,3,3 and 6 doses respectively (Table 3). All four study patients tolerated the treatment protocol well. Minor adverse effects were experienced, including Grade 1 dizziness, mild abdomen tenderness, and mild headache. No serious adverse event was reported. At the conclusion of treatment, all subjects reported a general state of well-being, that they were feeling very good. There were no abnormal test results that warranted additional diagnostic testing, medical or surgical intervention, dose adjustment or use of an additional drug treatment. One patient who was withdrawn prior to completion of the study had an abnormal serum chemistry of elevated potassium level and high creatinine level reflecting a possible underlying renal impairment.

\begin{tabular}{|c|c|c|c|c|}
\hline Patient & Cancer & $\begin{array}{c}\text { Pretest } \\
\text { Platelet Count } \\
\text { (cells/ul) }\end{array}$ & $\begin{array}{c}\text { Posttest } \\
\text { Platelet Count } \\
\text { (cells/ul) }\end{array}$ & $\begin{array}{c}\text { \# of Test } \\
\text { Doses } \\
\text { Received }\end{array}$ \\
\hline 1 & Breast & 34,000 & 110,000 & 3 \\
\hline 2 & Ovarian & 42,000 & 92,000 & 3 \\
\hline 3 & Prostate & 39,000 & 110,000 & 3 \\
\hline 4 & Colon & 33,000 & 220,000 & 6 \\
\hline
\end{tabular}

Table 3: Results of Test Administration

\section{Discussion}

Oprelvekin stimulates megakaryocytopoesis thru the interleukin-11 pathway as outlined in Figures 1 and 2 [8,16]. In 1996 Kaye $(3,4)$ conducted the first study of the use of recombinant human interleukin 11 (rhIL-11) in severe chemotherapy-induced thrombocytopenia who those receiving a variety of chemotherapeutic regimens for breast cancer, solid tumors and lymphomas. The recombinant human interleukin-11 prevented the need for platelet transfusions in as much as $53 \%$ of patients in subsequent chemotherapeutic cycles [17]. Chemotherapy-induced mucositis was also ameliorated [3,4]. Thus, the role of rhIL-11 in thrombocytopenia-induced chemotherapy is invaluable. Prolonged chemotherapeutic cycles in malignancies have inherent side effects and adverse events. As such, additional treatment modalities employed to treat these complications have to be evaluated in the light of their compounding effect(s). However it has been demonstrated that despite the described adverse events, chemotherapy has continued at full doses without interruption [17].

At the time of US FDA approval in 1997, Oprelvekin was noted to incur tachycardia in patients $[6,13,14]$. However, the ability of the drug to improve platelet counts overshadows its mild cardiac effects. In previous clinical trials $[7,11,18]$ atrial flutter or fibrillation occurred in $12-15 \%$ of patients treated with the dose of $50 \mathrm{mcg} / \mathrm{kg}$. The arrhythmias were brief, underwent spontaneous (or after test dose rate control) cardioversion to normal sinus rhythm without clinical sequelae. The mechanism of arrhythmia development is unknown but is postulated to be from increased plasma volume with fluid retention [8,19,20]. However four risk factors are associated with atrial arrhythmias: age, prior history of arrhythmias, cardiac disorder, and alcohol use [9]. Agedependent increased atrial dimension and reduced atrial refractoriness have been described in old rats treated with rhIL-11 [21]. Sodium retention producing atrial flutter or fibrillation was postulated as the most likely mechanism producing this effect. Studies on ventricular function have not been described to date.

It was concluded that the common adverse effects of Oprelvekin should not deter the use of the drug. Moreover, the side effects are relatively mild, non-toxic, and can be easily managed $[19,22]$. The risk for stroke is increased in those with prior history or those experiencing transient ischemic attacks [11]. But these are rare and would necessitate diligent medical screening.

All four study patients had 12-lead ECG readings at baseline and all throughout the study protocol. All the ECG readings were interpreted as normal. The study drug was well tolerated. The thrombocytopenia induced from chemotherapy resolved without the need for platelet transfusion for thrombocytopenia (platelet counts $<50,000$ cells /ul). The clinical evidence supports the role of Oprelvekin in megakaryocytopoiesis as it accelerates platelet recovery as outlined [9]. It remains the primary treatment of choice for the prevention of severe thrombocytopenia (platelet counts $<20,000$ cells/ul) following myelosuppressive chemotherapy in patients with nonmyeloid malignancies $[3,4,7]$. All patients were at high risk for severe thrombocytopenia but they responded to the study protocol with platelet counts that did not go below the level that would have required platelet transfusion. As such potential complications from platelet transfusions such as infections, reduction in patient quality of life and delayed chemotherapy were prevented [9]. In a study of early cirrhotic patients, Oprelvekin not only improved platelet counts but actually decreased significantly liver enzymes [12]. This finding suggested that IL-11 blocks activation of proinflammatory cytokines and downregulates tumor necrosis alpha factor [12]. This finding is interesting as it may potentiate the beneficial effects of the patient's own chemotherapeutic regimen. Further studies would need to be conducted. 


\section{Conclusion}

Oprelvekin did not produce any detrimental cardiac effects and should be continued as a suitable treatment for chemotherapyinduced thrombocytopenia. However, given the limited sample size, the effects of Oprelvekin in cardiac repolarization is not generalizable to all patients suffering from thrombocytopenia. Cancer treatments are incredibly complex; the effects of Oprelvekin may differ from patient to patient depending on the nature and stage of their malignancy as well as to the type of chemotherapy regimen they undertake. However, all four patients in this phase 2 clinical trial had varying types of malignancy and were at different cycles of their chemotherapeutic regimens. No patient demonstrated any cardiac complications after subcutaneous 50 $\mathrm{mcg} / \mathrm{kg}$ of Oprelvekin for thrombocytopenia platelet count $<50,000$ cells/ul. The demonstrated efficacy and tolerance in this small cohort of non-myeloid carcinoma patients sheds light on its potential usefulness in longer chemotherapeutic protocols and its ability to prevent platelet transfusions in multiple cycles of chemotherapy.

\section{Ethical Consideration}

This study was conducted in accordance with applicable laws and regulations including, but not limited to, the International Conference on Harmonisation (ICH), Guideline for Good Clinical Practice (GCP), and the ethical principles that have their origins in the Declaration of Helsinki. The institutional review board (IRB) reviewed and approved the protocol and informed consent form (ICF) before any subjects were enrolled. Before any protocol-required procedures were performed, the subjects signed and date the IRB-approved ICF.

\section{Acknowledgement}

Research facility provided by Envision Clinical Research, LLC 401 Westmont Laredo, Texas 78041.

\section{References}

1. Du X, Williams DA (1997) Interleukin-11: Review of Molecular, Cell Biology, and Clinical Use. Blood 89: 3897-908.

2. Kuter DJ (2015) Managing thrombocytopenia associated with cancer chemotherapy. Oncology 29: 282-94.

3. Kaye JA (1996) The clinical development of recombinant human interleukin 11 (NEUMEGA rhIL-11 growth factor). Stem Cells 14: 256-60.

4. Kaye JA (1998) FDA licensure of NEUMEGA to prevent severe chemotherapy-induced thrombocytopenia. Stem Cells 16: 207-23.

5. Liebman HA (2014) Thrombocytopenia in cancer patients. Thromb Res 133: S63-9.

6. Pfizer (2016) NEUMEGA (oprelvekin), USA.

7. Tepler I, Elias L, Smith JW 2nd, Hussein M, Rosen G, et al. (1996) A randomized placebo-controlled trial of recombinant human interleukin-11 in cancer patients with severe thrombocytopenia due to chemotherapy. Blood 87: 3607-14.

8. Davis MR, Zhu Z, Hansen DM, Bai Q, Fang Y (2015) The role of IL-21 in immunity and cancer. Cancer letters 358: 107-14.

9. Smith JW (2000) Tolerability and side-effect profile of rhIL-11. Oncology 14: 41-7.

10. Food and Drug Administration (2016) Therapeutic Biologics Applications (BLA), USA.

11. Wyeth Pharmaceuticals Inc. (1993) NEUMEGA [nu-meg<a] (oprelvekin), Philadephia, PA, USA.

12. Ghalib R, Levine C, Hassan M, McClelland T, Goss J, et al. (2003) Recombinant human interleukin-11 improves thrombocytopenia in patients with cirrhosis. Hepatology 37: 1165-71.

13. Pfizer (2016) NEUMEGA [nu-meg<a] (oprelvekin), USA.

14. Wyeth Pharmaceuticals (2016) NEUMEGA- oprelvekin, Wyeth BioPharma Division of Wyeth Pharmaceuticals Inc., a subsidiary of Pfizer Inc., USA.

15. Crediblemeds (2017) QTDrugs List, USA.

16. Nandurkar HH, Robb L, Begley CG (1998) The role of IL-11 in hematopoiesis as revealed by a targeted mutation of its receptor. Stem Cells 16: 53-65.

17. Reynolds CH (2000) Clinical efficacy of rhIL-11. Oncology 14: 32-40.

18. Isaacs C, Robert NJ, Bailey FA, Schuster MW, Overmoyer B, et al. (1997) Randomized placebo-controlled study of recombinant human interleukin-11 to prevent chemotherapy-induced thrombocytopenia in patients with breast cancer receiving dose-intensive cyclophosphamide and doxorubicin. J Clin Oncol 15: 3368-77.

19. Baldo BA (2014) Side effects of cytokines approved for therapy. Drug Saf 37: 921-43.

20. Wilde M, Faulds D (1998) Oprelvekin, A Review of its Pharmacology and Therapeutic Potential in Chemotherapy-indced Thrombocytopenia. BioDrugs 10: $159-71$.

21. Xu J, Ren JF, Mugelli A, Belardinelli L, Keith Jr JC Jr, et al. (2002) Age-dependent atrial remodeling induced by recombinant human interleukin-11: implications for atrial flutter/fibrillation. J Cardiovasc Pharmacol 39: 435-40.

22. Gordon MS, McCaskill-Stevens WJ, Battiato LA, Loewy J, Loesch D, et al. (1996) A phase I trial of recombinant human interleukin-11 (neumega rhIL-11 growth factor) in women with breast cancer receiving chemotherapy. Blood 87: 3615-24. 


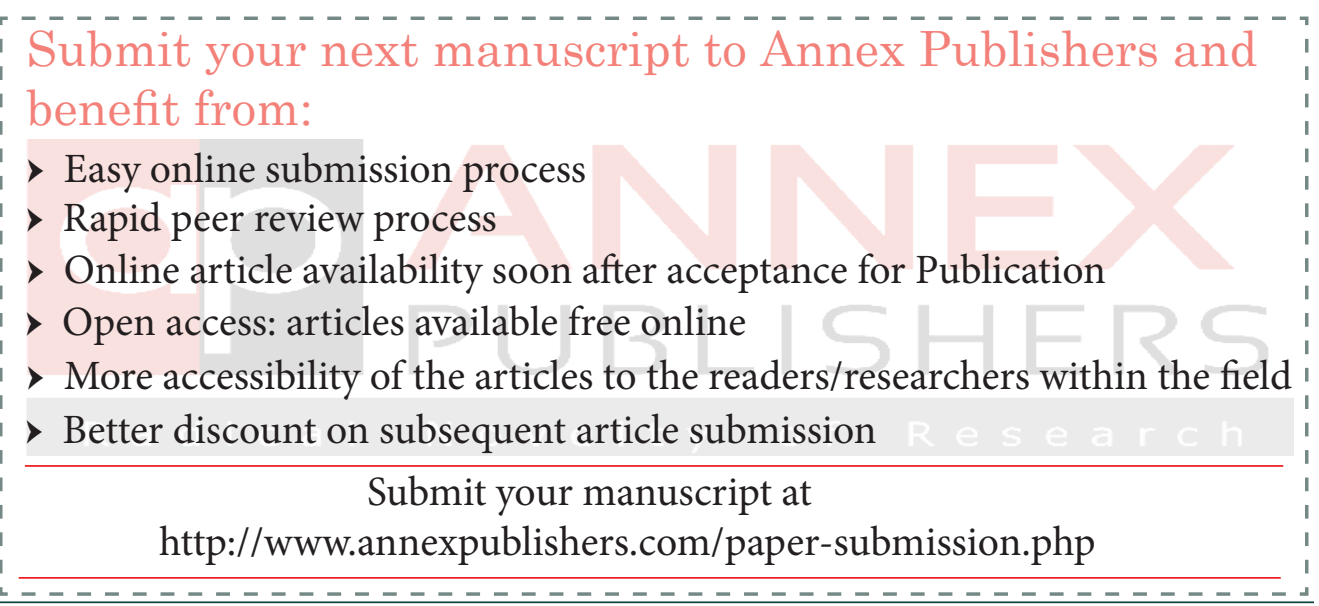

